\title{
ON THE SOLUTION OF EQUATIONS WITH NONDIFFERENTIABLE OPERATORS
}

\author{
IOANNIS K. ARGYROS
}

\begin{abstract}
A bstract. We approximate solutions of equations with nondifferentiable operators using the Newton-Kantorovich method and the majorant theory. Under some as easy to verify assumptions as the ones given by Zabrejko and Nguen in [9] we improve their error estimates.
\end{abstract}

\section{Introduction}

Let $X$ and $Y$ be Banach spaces, and let $U\left(x_{0}, R\right)$ denote the closed ball with center $x_{0} \in X$ and of radius $R$ in $X$. Suppose that two operators $F$ and $G$ are defined on a convex subset $D$ of $X$ containing $U\left(x_{0}, R\right)$, with values in $Y$, where $F$ is Fréchet differentiable at every interior point of $U\left(x_{0}, R\right)$ and satisfies the condition

$$
\left\|F^{\prime}(x+h)-F^{\prime}(x)\right\| \leq A(r,\|h\|), x \in U\left(x_{0}, r\right), 0 \leq r \leq R, 0 \leq\|h\| \leq R-r,
$$

while $G$ satisfies the condition

$$
\|G(x+h)-G(x)\| \leq B(r,\|h\|), x \in U\left(x_{0}, r\right), 0 \leq r \leq R, 0 \leq\|h\| \leq R-r .
$$

Here $A, B$ are nonnegative and continuous functions of two variables such that if one of the variables is fixed then $A, B$ are non-decreasing functions of the other on the interval $[0, R]$. Moreover, the following are true:

Received April 2, 1990.

(1980) A. M. S. classification codes: 49D15, 47H17, 65.

Keywords and phrases: Banach space, Newton's method, Banach's, closed graph theorem. 
(a) the function $\frac{\partial A(0, t)}{\partial t}$ is positive, continuous and non-decreasing on $[0, R-r]$ with $A(0,0)=0$;

(b) $B$ is linear in the second variable and the function $\frac{\partial B(R, t)}{\partial t}$ is positive, continuous and non-decreasing on $[0, R-r]$.

the definition of $B$ implies that

$$
B(r, t) \leq B(R, t), \text { for all } 0 \leq r \leq R \text { and } 0 \leq t \leq R-r
$$

Further, assume that the operator $F^{\prime}\left(x_{0}\right)$ is invertible. We are concerned with approximating a solution $x^{*}$ of the equation

$$
F(x)+G(x)=0
$$

in $U\left(x_{0}, R\right)$ using the approximations

$$
z_{n+1}=z_{n}-F^{\prime}\left(x_{0}\right)^{-1}\left(F\left(z_{n}\right)+G\left(z_{n}\right)\right), z_{0}=x_{0}, n=0,1,2, \cdots
$$

and

$$
x_{n+1}=x_{n}-F^{\prime}\left(x_{n}\right)^{-1}\left(F\left(x_{n}\right)+G\left(x_{n}\right)\right), \quad n=0,1,2, \cdots .
$$

Equation (5) has been studied extensively in the case when $G=0$, using the modified iteration (6) or the Newton-Kantorovich iteration (7), [2], [3], [4], [5]. In particular, Potra and Ptâk have obtained elegant error estimates by means of a method based on a special variant of Banach's closed graph theorem [1], [4], [8]. Zincenko in [10] and Zabrejko and Ngyen in [9] studied the case $G \neq 0$ under the hypotheses (1) and (2) provided that $A, B$ are given by

$$
A(r, t)=k(r) t
$$

and

$$
B(r, t)=\epsilon(r) t
$$

where $k(r)$ and $\epsilon(r)$ are non-decreasing functions on the interval $[0, R]$. Further work on equation (5) can be found in [8]. 
In this paper we show that under very similar assumptions our error estimates on the distances $\left\|x^{*}-x_{n}\right\|,\left\|x_{n}-x_{n+1}\right\|\left(\left\|x^{*}-z_{n}\right\|,\left\|z_{n}-z_{n+1}\right\|\right)$ are better than the ones given in [9].

We will assume that for a fixed $r \in[0, R]$ the functions $A$ and $B$ can be extended such that $\|h\| \in[0, r]$.

\section{Existence Theorems}

We will need to define the constants

$$
a=\left\|F^{\prime}\left(x_{0}\right)^{-1}\left(F\left(x_{0}\right)+G\left(x_{0}\right)\right)\right\|, \quad b=\left\|F^{\prime}\left(x_{0}\right)^{-1}\right\|
$$

and the functions

$$
\begin{aligned}
\omega(r) & =\int_{0}^{r} k(t) d t \\
\varphi(r) & =a+b \int_{0}^{r} \omega(t) d t-r \\
\varphi_{\gamma}(r) & =a+b \int_{0}^{r} A(0, t) d t-r \\
\psi(r) & =b \int_{0}^{r} \epsilon(t) d t \\
\psi_{\gamma}(t) & =b B(R, t) \\
\chi(r) & =\varphi(r)+\psi(r)
\end{aligned}
$$

and

$$
\chi_{\gamma}(r)=\varphi_{\gamma}(r)+\psi_{\gamma}(r) .
$$

As in [9], the main advantage of our approach consists in the fact that the study of equation (5) reduces to the study of a simple scalar equation

$$
\chi_{\gamma}(r)=0 \text { on }[0, R]
$$

The following theorem justifies our claim.

Theorem 1. Suppose that the function $\chi_{\gamma}(r)=\varphi_{\gamma}(r)+\psi_{\gamma}(r)$ has a unique zero $s$ in the interval $[0, R]$, and $\chi_{\gamma}(R) \leq 0$. Then equation (5) has a solution 
$x^{*}$ in $U\left(x_{0}, r\right)$. This solution is unique in $U\left(x_{0}, s\right)$, and the iterates generated by

(6) are well-defined for all $n$, belong to $U\left(x_{0}, s\right)$, and satisfy the estimates

$$
\left\|z_{n+1}-z_{n}\right\| \leq \bar{s}_{n+1}-\bar{s}_{n}, \quad n=0,1,2, \cdots
$$

and

$$
\left\|z_{n}-x^{*}\right\| \leq s-\bar{s}_{n}, \quad n=0,1,2, \cdots .
$$

Moreover, the sequence defined by

$$
\bar{s}_{n+1}=d\left(\bar{s}_{n}\right), \quad n=0,1,2, \cdots, \bar{s}_{0}=0
$$

where

$$
d_{\gamma}(r)=r+\chi_{\gamma}(r)
$$

is monotonically increasing and converges to $s$.

Proof. Let us define the sequence

$$
z_{n+1}=P\left(z_{n}\right), \quad n=0,1,2, \cdots, z_{0}=x_{0}
$$

where

$$
P(z)=I-F^{\prime}\left(x_{0}\right)^{-1}(F(z)+G(z)) .
$$

Moreover, consider the numerical sequence given by (21).

As in $[9$, p.675], a simple geometrical argument shows that the sequence (21) is monotonically increasing and convergent to $s$.

We shall show the estimate

$$
\left\|z_{n+1}-z_{n}\right\| \leq \bar{s}_{n+1}-\bar{s}_{n}
$$

For $n=0$ the inequality (25) is true since

$$
\left\|z_{1}-z_{0}\right\|=\left\|F^{\prime}\left(x_{0}\right)^{-1}\left(F\left(x_{0}\right)+G\left(x_{0}\right)\right)\right\|=a=d(0)=\bar{s}_{1}-\bar{s}_{0} .
$$

Let us assume that (25) holds for $n=0,1,2, \cdots, k-1$. Then

$$
\begin{aligned}
& \left\|z_{k+1}-z_{k}\right\|=\left\|P\left(z_{k}\right)-P\left(z_{k-1}\right)\right\| \\
\leq & \left\|z_{k}-z_{k-1}-F^{\prime}\left(x_{0}\right)^{-1}\left(F\left(z_{k}\right)-F\left(z_{k-1}\right)\right)\right\|+\left\|F^{\prime}\left(x_{0}\right)^{-1}\left(G\left(z_{k}\right)-G\left(z_{k-1}\right)\right)\right\| \\
\leq & b \int_{0}^{1}\left\|F^{\prime}\left((1-t) z_{k-1}+t z_{k}\right)-F^{\prime}\left(x_{0}\right)\right\|\left\|z_{k}-z_{k-1}\right\| d t+b\left\|G\left(z_{k}\right)-G\left(z_{k-1}\right)\right\| .
\end{aligned}
$$


Set

$$
h=(1-t) z_{k-1}+t z_{k}-(1-t) x_{0}-t x_{0}
$$

then

$$
\|h\| \leq(1-t)\left\|z_{k-1}-x_{0}\right\|+t\left\|z_{k}-x_{0}\right\| \leq(1-t) \bar{s}_{k-1}+t \bar{s}_{k} .
$$

Using (1), (2), (4), (26), (27) and the induction hypothesis, (26) becomes

$$
\begin{gathered}
\left\|z_{k+1}-z_{k}\right\| \leq b \int_{\bar{s}_{k-1}}^{\bar{s}_{k}} A(0, t) d t+b B\left(\bar{s}_{k-1},\left\|z_{k}-z_{k-1}\right\|\right) \\
\leq b\left[\int_{\bar{s}_{k-1}}^{\bar{s}_{k}} A(0, t) d t+b B\left(R, \bar{s}_{k}-\bar{s}_{k-1}\right)\right]=d_{\gamma}\left(\bar{s}_{k}\right)-d_{\gamma}\left(\bar{s}_{k-1}\right)=\bar{s}_{k+1}-\bar{s}_{k},
\end{gathered}
$$

which shows (25) for $n=k$.

We have shown that (21) is a Cauchy sequence in a Banach space and as such it converges to a solution $x^{*} \in U\left(x_{0}, s\right)$. We will now show that $x^{*}$ is a unique solution of equation (5) in $U\left(x_{0}, s\right)$. Let us define the sequences

$$
y_{n+1}=P\left(y_{n}\right), \quad n=0,1,2, \cdots y_{0} \in U\left(x_{0}, \mathbb{R}\right)
$$

and

$$
s_{n+1}^{+}=d_{\gamma}\left(s_{n}^{+}\right), \quad n=0,1,2, \cdots, \quad s_{0}^{+}=R
$$

To show uniqueness it suffices to show the estimates

$$
\left\|z_{n}-y_{n}\right\| \leq s_{n}^{+}-s_{n}^{-}, \quad n=0,1,2, \cdots
$$

Using the same geometrical argument as in $[9$, p. 675] we can show that the sequence (29) is monotonically decreasing and convergent to $s$. That is, if for $y_{0}$ we choose the second solution $x_{1}^{*} \in U\left(x_{0}, r\right)$ of equation (5), we get by (30), that $\left\|x_{1}^{*}-x^{*}\right\| \leq s_{n}^{+}-s_{n}^{-}$. That is, $x_{1}^{*}=x^{*}$. For $n=0,(30)$ is true since $\left\|y_{0}-z_{0}\right\| \leq \mathbb{R}-0=R$. Let us assume that (30) holds for $n=0,1,2, \cdots, k$. 
Using (1) and (2)

$$
\begin{aligned}
& \left\|y_{k+1}-z_{k+1}\right\|=\left\|p\left(y_{k}\right)-P\left(z_{k}\right)\right\| \\
\leq & \left\|y_{k}-z_{k}-F^{\prime}\left(x_{0}\right)^{-1}\left(F\left(y_{k}\right)-F\left(z_{k}\right)\right)\right\|+\left\|F^{\prime}\left(x_{0}\right)^{-1}\left(G\left(y_{k}\right)-G\left(z_{k}\right)\right)\right\| \\
\leq & b \int_{0}^{1}\left\|F^{\prime}\left((1-t) z_{k}+t y_{k}\right)-F^{\prime}\left(x_{0}\right)\right\| \cdot\left\|y_{k}-z_{k}\right\| d t+b B\left(R, s_{k}^{+}-s_{k}^{-}\right) \\
\leq & b \int_{0}^{1} A\left(0,(1-t) s_{k}^{-}+t s_{k}^{+}\right)\left(s_{k}^{+}-s_{k}^{-}\right) d t+b B\left(R, s_{k}^{+}-s_{k}^{-}\right) \\
\leq & b \int_{s_{k}^{-}}^{s_{k}^{+}} A(0, t) d t+b B\left(R, s_{k}^{+}-s_{k}^{-}\right)=d_{\gamma}\left(s_{k}^{+}\right)-d_{\gamma}\left(s_{k}^{-}\right)=s_{k+1}^{+}-s_{k+1}^{-} .
\end{aligned}
$$

That completes the proof of the theorem.

We can now prove the main theorem.

Theroem 2. Assume

(i) the hypotheses of Theorem 1 are ture;

(ii) the number $R$ defined in Theorem 1 is such that

$$
1-b A(0, R)>0
$$

and

$$
T(R) \leq R
$$

where

$$
T(r)=a+\frac{b \int_{0}^{r} A(r, t) d t+b B(r, r)}{1-b A(0, r)}, \quad r \in[0, \infty) .
$$

Then

(a) the sequence $\left\{\bar{\rho}_{n}\right\}, n=0,1,2, \cdots$ given by

$$
\begin{gathered}
\bar{\rho}_{0}=0, \quad \bar{\rho}_{1}=a, \\
\bar{\rho}_{n+1}=\bar{\rho}_{n}-\frac{b \int_{\bar{\rho}_{n-1}}^{\bar{\rho}_{n}} A\left(\bar{\rho}_{n-1}, t\right) d t+\psi_{\gamma}\left(\bar{\rho}_{n}\right)-\dot{\psi}_{\gamma}\left(\bar{\rho}_{n-1}\right) .}{\varphi_{\gamma}^{\prime}\left(\bar{\rho}_{n}\right)}, \quad n=1,2, \cdots
\end{gathered}
$$


is bounded above by $R$, it is monotonically increasing and converges to some $s^{*}$ with $s \leq s^{*} \leq R$. The sequence $\left\{q_{n}\right\}$ given by

$$
q_{n+1}=q_{n}-\frac{\chi_{\gamma}\left(q_{n}\right)}{\varphi_{\gamma}^{\prime}\left(q_{n}\right)}, \quad q_{0}=0, \quad n=0,1,2, \cdots
$$

is monotonically increasing and converges to $s$. Moreover

$$
\begin{gathered}
q_{n+1}-q_{n} \leq \bar{\rho}_{n+1}-\bar{\rho}_{n}, \\
s-q_{n} \leq s^{*}-\bar{\rho}_{n}
\end{gathered}
$$

and

$$
q_{n} \leq \bar{\rho}_{n} \text { for all } n=0,1,2, \cdots .
$$

(b) The iterates generated by (7) are well-defined in $U\left(x_{0}, s^{*}\right)$ for all $n$, and satisfy the estimates

$$
\left\|x_{n+1}-x_{n}\right\| \leq \bar{\rho}_{n+1}-\bar{\rho}_{n}, \quad n=0,1,2, \cdots
$$

and

$$
\left\|x_{n+1}-x^{*}\right\| \leq s^{*}-\bar{\rho}_{n}, \quad n=0,1,2, \cdots
$$

Proof. (a) We will first show that the sequence given by (31) is bounded above by $R$. This is true for $k=0,1$. For $k=2$ we have

$$
\bar{\rho}_{2}=a+\frac{b \int_{0}^{a} A(0, t) d t+b B(R, a)}{1-b A(0, a)} \leq T(R) \leq R .
$$

Let us assume that $\bar{\rho}_{k} \leq R$ for $k=0,1,2, \cdots, n$. We will show that $\bar{\rho}_{k} \leq R$ for $k=n+1$. The sequence given by (31) is such that

$$
\begin{gathered}
\bar{\rho}_{n+1} \leq \bar{\rho}_{n}+\frac{b \int_{\bar{\rho}_{n-1}}^{\bar{\rho}_{n}} A(R, t) d t+b B\left(R, \bar{\rho}_{n}-\bar{\rho}_{n-1}\right)}{1-b A(0, R)} \\
\leq \cdots \leq a+\frac{b\left[\int_{\bar{\rho}_{n-1}}^{\bar{\rho}_{n}} A(R, t) d t+\int_{\bar{\rho}_{1}}^{\bar{\rho}_{2}} A(R, t) d t+\cdots+\int_{\bar{\rho}_{n-1}}^{\bar{\rho}_{n}} A(R, t) d t\right]}{1-b A(0, R)} \\
\quad+\frac{b\left(B\left(R, \bar{\rho}_{1}-\bar{\rho}_{0}+\bar{\rho}_{2}-\bar{\rho}_{1}+\cdots+\bar{\rho}_{n}-\bar{\rho}_{n-1}\right)\right.}{1-b A(0, R)} \\
=T(R) \leq R, \text { for all } n=0,1,2, \cdots,
\end{gathered}
$$


by hypothesis. Using (31) it can easily be seen that the sequence $\left\{\bar{\rho}_{n}\right\}$ is monotonically increasing. Hence, it converges to some $s^{*}$ with $a \leq s^{*} \leq R$. The same proof as the proof of Proposition 3 in $\left[9\right.$, p.677] shows that the sequence $\left\{q_{n}\right\}$ is monotonically increasing and converges to $s$. It can easily be seen using induction on $n$ that $\varphi^{\prime}\left(q_{n}\right) \leq \varphi^{\prime}\left(\bar{\rho}_{n}\right)$ for all $n=0,1,2, \cdots$. Using the definitions of the sequences $\left\{q_{n}\right\}$ and $\left\{\bar{\rho}_{n}\right\}$ and the above inequality we can now easily deduce the rest of the results in (a).

(b) As in [9, p.678] for $n=0,(32)$ is true. Suppose that (32) is ture for $n=0,1,2, \cdots, k-1$. We must show that $F^{\prime}\left(x_{k}\right)$ is invertible. We have

$$
\left\|x_{k}-x_{0}\right\| \leq \sum_{j=1}^{k}\left\|x_{j}-x_{j-1}\right\| \leq \sum_{j=1}^{k}\left(\bar{\rho}_{j}-\bar{\rho}_{j-1}\right)=\bar{\rho}_{k}
$$

(by the induction hypothesis).

By (1) we obtain

$$
\left\|F^{\prime}\left(x_{0}\right)^{-1}\left(F^{\prime}\left(x_{k}\right)-F^{\prime}\left(x_{0}\right)\right)\right\| \leq b A\left(0, \bar{\rho}_{k}\right)<b A\left(0, s^{*}\right)=\varphi_{\gamma}^{\prime}\left(s^{*}\right)+1 \leq 1 .
$$

By the Banach lemma on invertible operators $F^{\prime}\left(x_{k}\right)$ is also invertible and

$$
\left\|F^{\prime}\left(x_{k}\right)^{-1}\right\| \leq \frac{b}{1-b A\left(0, \bar{\rho}_{k}\right)}=-\frac{b}{\varphi_{\gamma}^{\prime}\left(\bar{\rho}_{k}\right)} .
$$

Using (7) we get

$$
\begin{aligned}
\left\|x_{k+1}-x_{k}\right\| & =\left\|F^{\prime}\left(x_{k}\right)^{-1}\left(F\left(x_{k}\right)+G\left(x_{k}\right)\right)\right\| \\
= & \| F^{\prime}\left(x_{k}\right)^{-1}\left(F\left(x_{k}\right)-F\left(x_{k-1}\right)-F^{\prime}\left(x_{k-1}\left(x_{k}-x_{k-1}\right)+G\left(x_{k}\right)-G\left(x_{k-1}\right)\right) \|\right. \\
& \leq\left\|F^{\prime}\left(x_{k}\right)^{-1}\right\|\left[\int_{0}^{1}\left\|F^{\prime}\left((1-t) x_{k-1}+t x_{k}\right)-F^{\prime}\left(x_{k-1}\right)\right\| \cdot\left\|x_{k}-x_{k-1}\right\| d t\right. \\
& \left.\quad+\left\|G\left(x_{k}\right)-G\left(x_{k-1}\right)\right\|\right] .
\end{aligned}
$$

By (1), (2), (34) and (35), (36) becomes,

$$
\begin{aligned}
\left\|x_{k+1}-x_{k}\right\| \leq & -\frac{b}{\varphi_{\gamma}^{\prime}\left(\bar{\rho}_{k}\right)} \int_{0}^{1} A\left(\bar{\rho}_{k-1},(1-t) \bar{\rho}_{k-1}+t \bar{\rho}_{k}\right)\left(\bar{\rho}_{k}-\bar{\rho}_{k-1}\right) d t \\
& -\frac{1}{\varphi_{\gamma}^{\prime}\left(\bar{\rho}_{k}\right)}\left(\varphi_{\gamma}\left(\bar{\rho}_{k}\right)-\psi_{\gamma}\left(\bar{\rho}_{k-1}\right)\right) \\
= & -\frac{b}{\varphi_{\gamma}^{\prime}\left(\bar{\rho}_{k}\right)} \int_{\bar{\rho}_{k-1}}^{\bar{\rho}_{k}} A\left(\bar{\rho}_{k-1}, t\right) d t-\frac{1}{\varphi_{\gamma}^{\prime}\left(\bar{\rho}_{k}\right)}\left(\psi_{\gamma}\left(\bar{\rho}_{k}\right)-\psi_{\gamma}\left(\bar{\rho}_{k-1}\right)\right) \\
= & \bar{\rho}_{k+1}-\bar{\rho}_{k} .
\end{aligned}
$$


That shows (32) for $n=k$. The estimates (33) follow now immediately using (32).

That completes the proof of the theorem.

In Theorem 2 we really wanted to show that the sequence $\left\{q_{n}\right\}$ majorizes the sequence $\left\{x_{n}\right\}$. Instead we showed the results for the sequence $\left\{\bar{\rho}_{n}\right\}$.

The corresponding estimates to (19), (20), (32) and (33) in [9] are given by

$$
\begin{aligned}
\left\|z_{n+1}-z_{n}\right\| & \leq \rho_{n+1}^{*}-\rho_{n}^{*} \\
\left\|z_{n}-z^{*}\right\| & \leq \rho-\rho_{n}^{*} \\
\left\|x_{n+1}-x_{n}\right\| & \leq \rho_{n+1}-\rho_{n}
\end{aligned}
$$

and

$$
\left\|x_{n}-x^{*}\right\| \leq \rho-\rho_{n}
$$

where $\rho$ is the unique zero of $\chi(r)=\varphi(r)+\psi(r)$ in $[0, R]$ and the sequences $\left\{\rho_{n}^{*}\right\}$ and $\left\{\rho_{n}\right\}$ are given by

$$
\rho_{n+1}^{*}=d\left(\rho_{n}^{*}\right), \quad n=0,1,2, \cdots, \rho_{0}^{*}=0,
$$

where

$$
d(r)=r+\chi(r)
$$

and

$$
\rho_{n+1}=\rho_{n}-\frac{\chi\left(\rho_{n}\right)}{\varphi^{\prime}\left(\rho_{n}\right)}, \quad n=0,1,2, \cdots, \rho_{0}=0 .
$$

We will now show that under simple assumptions

$$
\bar{\rho}_{n+1}-\bar{\rho}_{n} \leq \rho_{n+1}-\rho_{n} .
$$

We can show similiarly that the rest of our error estimates (19), (20) and (33) are better than (37), (38) and (40) respectively.

We will first need to state a theorem whose proof can be found in [9, p.673].

Theorem 3. Suppose that the function $\chi(r)=\varphi(r)+\psi(r)$ has a unique zero $\rho$ in the interval $[0, R]$, and $\chi(R) \leq 0$. Then equation (5) admits a solution $x^{*}$ in 
Proof. (a) We will only show (48). The rest will follow similarly. By (12)-(17), (44) and (45) we obtain immediately that

$$
\chi_{\gamma}(r) \leq \chi(r) \text { for all } r \in[0, R] .
$$

Moreover, using (44) we get

$$
-\varphi^{\prime}(r)=1-b \omega(r) \geq 1-b A(0, r)>0 .
$$

That is

$$
-\frac{1}{\varphi^{\prime}(r)} \leq-\frac{1}{\varphi_{\gamma}^{\prime}(r)}
$$

The results now follows from (55), (57), (31) and (43).

(b) By Theorems 2 and 3 to show (53) we only need to show

$$
\bar{\rho}_{n+1}-\bar{\rho}_{n} \leq \rho_{n+1}-\rho_{n} \text { for all } n=0,1,2, \cdots .
$$

By (41) and (43), inequality (58) will be true if

$$
\begin{gathered}
b \int_{\bar{\rho}_{n-1}}^{\bar{\rho}_{n n}} A\left(\bar{\rho}_{n-1}, t\right) d t+b B\left(R, \bar{\rho}_{n}-\bar{\rho}_{n-1}\right) \leq b \int_{0}^{\rho_{n}} \omega(t) d t+a-\rho_{n}+b \int_{0}^{\rho_{n}} \epsilon(t) d t \\
\text { for all } n=1,2, \cdots
\end{gathered}
$$

and

$$
A\left(0, \bar{\rho}_{n}\right) \leq \omega\left(\rho_{n}\right), \quad \text { for all } n=0,1,2, \cdots .
$$

The left hand side of (59) is bounded aobve by the left hand side of (51) for $n=1$ and by the left hand side of (52) for $n \geq 2$. Whereas the right hand side of (50) is bounded below by the right hand side of (51) for $n=1$ and the right hand side of (52) for $n \geq 2$. Hence (50) is true for all $n=1,2, \cdots$. Inequality (60) is certainly ture if inequality (50) is ture. That shows (53). Inequality (54) now follows easily. That completes the proof of the proposition.

Note that by Theorem $2, s^{*}$ can be replace with $R$ in (50)-(52).

A discussion on the convergence speed of the numerical sequences (21) and (31) can now easily follow as the discussion in [9, p. 679-684]. However, we leave that to the motivated reader. 
the ball $U\left(x_{0}, \rho\right)$, this solution is unique in $U\left(x_{0}, R\right)$, and the iterates generated by (6) and (7) are defined for all $n$, belong to $U\left(x_{0}, \rho\right)$ and satisfy the estimates (37)-(40). Moreover, the sequences $\left\{\rho_{n}^{*}\right\}$ and $\left\{\rho_{n}\right\}$ are monotonically increasing and convergent to $\rho$.

We can now justify the claim made at the introduction.

Proposition. Under the hypotheses of Theorems 2 and 3 ,

(a) If

$$
A(0, t) \leq \omega(t), \quad 0 \leq t \leq R-r
$$

and

$$
\psi_{\gamma}(r) \leq \psi(r), \quad 0 \leq r \leq R
$$

Then

$$
\begin{aligned}
\left\|z_{n+1}-z_{n}\right\| & \leq \bar{s}_{n+1}-\bar{s}_{n} \leq \rho_{n+1}^{*}-\rho_{n}^{*} \\
\left\|z_{n}-z^{*}\right\| & \leq s-\bar{s}_{n} \leq \rho-\rho_{n}^{*} \\
\bar{q}_{n+1}-q_{n} & \leq \rho_{n+1}-\rho_{n}
\end{aligned}
$$

and

$$
s-q_{n} \leq \rho-\rho_{n} \text { for all } n=0,1,2, \cdots
$$

(b) If

$$
\begin{aligned}
A\left(0, s^{*}\right) & \leq \omega(a) \\
b \int_{0}^{a} A(0, t) d t+b B(R, a) & \leq b \int_{0}^{a} \omega(t) d t+b \int_{0}^{a} \epsilon(t) d t
\end{aligned}
$$

and

$$
b \int_{a}^{s^{*}} A\left(s^{*}, t\right) d t+b B\left(R, s^{*}-a\right) \leq b \int_{0}^{\rho_{2}} \omega(t) d t+a-\rho+b \int_{0}^{\rho_{2}} \in(t) d t .
$$

Then

$$
\left\|x_{n+1}-x_{n}\right\| \leq \bar{\rho}_{n+1}-\bar{\rho}_{n} \leq \rho_{n+1}-\rho_{n}
$$

and

$$
\left\|x_{n}-x^{*}\right\| \leq s^{*}-\bar{\rho}_{n} \leq \rho-\rho_{n} \quad \text { for all } n=0,1,2, \cdots
$$


Remark. Let us define the function $\bar{\chi}_{\gamma}(r)$ by

$$
\bar{\chi}_{\gamma}(r)=b \int_{0}^{r} A(r, t) d t+b B(R, r)+(a-r)(1-b A(0, r))
$$

and the sequence $\left\{v_{n}\right\}$, by

$$
v_{n+1}=v_{n}-\frac{\bar{\chi}_{\gamma}\left(v_{n}\right)}{\varphi_{\gamma}^{\prime}\left(v_{n}\right)}, \quad v_{0}=0, \quad n=1,2, \cdots
$$

Then, under the hypotheses of Theorem $2, \bar{\chi}_{\gamma}(0)=a>0$ and $\bar{\chi}_{\gamma}(R) \leq 0$. That is, there exists a solution $s_{1}^{*} \in(0, R]$ of the equation $\bar{\chi}_{\gamma}(r)=0$. Using induction on $n$, it is simple calculus to show that the seqence $\left\{v_{n}\right\}$, is monotonically increasing and converges to $s_{1}^{*}$. Moreover,

$$
\begin{gathered}
\bar{\rho}_{n} \leq v_{n} \\
\left\|x_{n+1}-x_{n}\right\| \leq v_{n+1}-v_{n} \\
\left\|x_{n}-x^{*}\right\| \leq s^{*}-\rho_{n} \leq s_{1}^{*}-v_{n}, \text { for all } n=0,1,2, \cdots
\end{gathered}
$$

and

$$
s^{*} \leq s_{1}^{*} .
$$

Furthermore, if the following conditions are satisfied

$$
\begin{gathered}
\rho-a \leq\left(v_{2}-a\right)(1-b A(0, R)), \\
A(r, t) \leq \omega(t)
\end{gathered}
$$

and

$$
\psi_{\gamma}(t) \leq \psi(t) \text { for all } r, t \in[0, R]
$$

then

$$
\begin{aligned}
v_{n+1}-v_{n} & \leq \rho_{n+1}-\rho_{n}, \\
v_{n} & \leq \rho_{n}
\end{aligned}
$$

and

$$
s_{1}^{*}-v_{n} \leq \rho-\rho_{n} \text { for all } n=0,1,2, \cdots
$$




\section{References}

[1] I. K. Argyros, "On Newton's method and nondiscrete mathematical induction", Bull. Austral. Math. Soc., 38 (1988), 131-140.

[2] M. Balazs and G. Goldner, "On the method of the cord and on a modification of it for the solution of nonlinear operator equations", Stud. Cerc. Mat., 20 (1968), 981-990.

[3] W. B. Gragg and R. A. Tapia, "Optimal error bounds for the Newton-Kantorovich Theorem", S.I.A.M. J. Numer. Anal., 11 (1974), 10-13.

[4] F. A. Potra and V. Ptâk, "Sharp error bounds for Newton's process", Numer. Math., 34 (1980), 63-72.

[5] W. C. Rheinboldt, "A unified convergence theory for a class of iterative processes", S.I.A.M. J. Numer. Anal., 5 (1968), 42-63.

[6] J. W. Schmidt, "Unter Fehrerschranker für regular-falsi-verfahren", Period. Math. Hung., 9 (1978), 241-247.

[7] T. Yamamoto, "A method for finding sharp error bounds for Newton's method under the Kantorovich assumptions", Numer. Math., 44 (1986), 203-220.

[8] ___ "A note on a posteriori error bound of Zabreiko and Nguen for Zincenko's iteration", Numer. Funct. Anal. and Optimiz., 9 (9 and 10), (1987), 987-994.

[9] P. P. Zabrejko and D. F. Nguen, "The majorant method in the theory of NewtonKantorovich approximations and the Ptâk error estimates", Numer. Funct. Anal. Optimiz., 9 (1987), 671-684.

[10] A. I. Zincenko, "Some approximate methods of solving equations with non-differentiable operators, (Ukrainian). Dopovidi Akad. Navk. Ukrain. RSR, (1963), 156-161.

Department of Mathematical Sciences, New Mexico State University, Las Cruces, NM 88003, U. S. A. 